\title{
Auxin Extraction and Purification Based on Recombinant Aux/IAA Proteins
}

\author{
Yi Su', Weigui Luo ${ }^{1}$, Xiaofei Chen¹, Huizhen Liu', Yueqing Hu', Wanhuang Lin ${ }^{1,2}$ and Langtao Xiao ${ }^{1,2^{*}}$
}

\begin{abstract}
Background: Indole-3-acetic acid (IAA) extraction and purification are of great importance in auxin research, which is a hot topic in the plant growth and development field. Solid-phase extraction (SPE) is frequently used for IAA extraction and purification. However, no IAA-specific SPE columns are commercially available at the moment. Therefore, the development of IAA-specific recognition materials and IAA extraction and purification methods will help researchers meet the need for more precise analytical methods for research on phytohormones.

Results: Since the AUXIN RESISTANT/INDOLE-3-ACETIC ACID INDUCIBLE (AuX/IAA) proteins show higher specific binding capability with auxin, recombinant IAA1, IAA7 and IAA28 proteins were used as sorbents to develop an IAA extraction and purification method. A GST tag was used to solidify the recombinant protein in a column. Aux/IAA proteins solidified in a column have successfully trapped trace IAA in aqueous solutions. The IAA7 protein showed higher IAA binding capability than the other proteins tested. In addition, expression of the IAA7 protein in Drosophila Schneider 2 (S2) cells produced better levels of binding than IAA7 expressed in E. coli.
\end{abstract}

Conclusion: This work validated the potential of Aux/IAA proteins to extract and purify IAA from crude plant extracts once we refined the techniques for these processes.

Keywords: IAA, Extraction and Purification, Binding Capability, Aux/IAA, SPE

\section{Background}

As one of the most important categories of phytohormones, auxin contributes to virtually all aspects of plant growth and development $[1,2]$. Thus, phytohormone quantification is very important. Currently, IAA is extracted from plant tissues and then highly purified using standardized protocols [3, 4]. Exposure to light, heat and oxygen can cause the degradation of IAA during sample preparation and purification, as IAA is not very stable in an aqueous environment. Thus, an IAA tracer labelled with radioactivity is used to estimate the amount of recovery and correct for any loss of IAA [5]. In the classical method, IAA extraction from plant tissues involves liquid nitrogen freezing/lyophilization, grinding/homogenization, treatment with organic solvents and the removal of solid impurities. IAA is a weak polar molecule. Thus, organic solvents with polarity close to IAA are the most effective

\footnotetext{
* Correspondence: Itxiao@hunau.edu.cn

${ }^{1}$ Hunan Provincial Key Laboratory of Phytohormones and Growth Development, Hunan Agricultural University, Changsha, China

${ }^{2}$ Hunan Co-Innovation Center for Utilization of Botanical Functional Ingredients, Changsha, China
}

for IAA extraction from plant tissues, as they result in greater efficiency and higher levels of extraction and recovery. However, a few recent studies used distilled water to extract IAA from plant samples [6]. Alternate types of extraction methods have been reported in previous studies that use various organic solvents, such as ethanol, methanol, acetonitrile, chloroform, acetone, propanol, ethyl acetate and acetic acid [7-12]. Most recently, precooled $80 \%$ methanol has become a universal extraction solvent for IAA and several other phytohormones because of its high efficiency during extraction and enhanced levels of recovery $[6,13]$.

Purification after extraction is critical because the complex metabolites in crude plant extracts can influence the accuracy of the analysis of IAA. To overcome the interference from other compounds in the extracts, a number of methods based on different principles were applied to the extraction and purification of IAA from plant tissue. Liquid-liquid extraction (LLE) and liquidliquid microextraction (LLME), hollow fibre-based liquid-liquid-liquid microextraction (HF-LLLME) and dispersive liquid-liquid microextraction (DLLME) have 
been used previously for IAA purification [14-20]. Solid-phase extraction (SPE) and antibody-based immune methods have recently become widely used to extract and purify IAA [6, 21-26]. Optimized solid-phase microextraction (SPME) and double-layered SPE (DL/SPE) resulted in a higher level of recovery and better ability to remove pigments $[27,28]$. SPE and SPME cartridges filled with matrix compound (sorbent) are used to extract and purify the target molecules from mixtures in the solution, as the sorbent can selectively bind certain molecule(s) based on an array of mechanisms, including adsorption, hydrogen bonding, polar and nonpolar interactions, cation/anion exchange and size exclusion [29]. Since modern SPE/SPME techniques are usually applied online coupled with HPLC, the current emphasis involves choosing various sorbents for trapping analytes [30, 31]. Many commercial SPE/SPME columns based on different sorbents have been widely used for high-throughput phytohormone extraction and purification from crude plant extracts [22, 23, 26]. However, commercial SPE columns for multiple phytohormones, such as Sep-Pak C18, Oasis HLB, Oasis MCX and Oasis MAX, are not designed specifically for phytohormones such as IAA. Thus, they are not highly selective. Recently, molecularly imprinted polymers (MIPs) and polymer monolith microextraction (PMME) were used to improve the specific recognition capability for phytohormones [32-34]. MIPs have been widely used to detect the presence of compounds in the environment because they offer advantages such as easy, cheap and rapid preparation along with high thermal and chemical stability [35-37]. Immunoaffinity SPE sorbents, also known as immunosorbents, are based upon molecular recognition using antibodies that offer higher affinity and selectivity for the target molecule (antigen). They are suitable for extraction and purification of a single analyte from complex biological and environmental aqueous samples. Moreover, immunoaffinity chromatography (IAC) and immunoaffinity gels (IAG) have been used to purify $\mathrm{ABA}$ and cytokinins [38-42].

Advances in phytohormonal research require greater efficiency and increased sensitivity for the analysis of phytohormones. This should lead to advances in phytohormone extraction and purification that occur more quickly in a less complicated manner. The techniques for sample preparation are complicated, and most methods are not specially designed for IAA. In the auxin signalling pathway, auxin action is based on binding to the TRANSPORT INHIBITOR RESPONSE1/AUXIN SIGNALING F-BOX (TIR1/AFB) nuclear receptors. Auxin stabilizes the co-receptor complex composed of the TIR1/AFB and AUXIN RESISTANT/INDOLE-3ACETIC ACID INDUCIBLE (Aux/IAA) proteins that trigger the proteasome-dependent degradation of the Aux/IAA transcriptional inhibitor to release the Auxin
Response Factor (ARF) factor that induces the auxinmediated transcriptional reprogramming [43, 44]. Interestingly, TIR1, Aux/IAA proteins and co-receptors (complex of TIR1/AFB and Aux/IAA) show high affinity towards auxin both in vivo and in vitro, as this hormone acts as the molecular glue that complexes the TIR1/AFB and Aux/IAA proteins [45]. Their auxin-specific binding characteristics can be utilized to improve the procedures for auxin extraction and purification. Therefore, we expressed AtIAA1, AtIAA7 and AtIAA28 in Escherichia coli and Drosophila Schneider 2 (S2) cells and developed a method for IAA extraction and purification using the recombinant proteins as the recognition molecules.

\section{Methods \\ Chemicals and Reagents}

GST Sefirose ${ }^{\mathrm{Tn}}$ resin, reduced glutathione and columns were purchased from the Shanghai Sangon Biotech Company (Shanghai, China). Enzymes, relative reagents and kits in gene cloning and vector construction were purchased from TransGen Biotech (Beijing, China). Stable isotope-labelled standard $\left[{ }^{2} \mathrm{H}_{5}\right]$ IAA was purchased from Olchemim Ltd. (Olomouc, Czech Republic). HPLC-grade acetonitrile (ACN) and methanol were obtained from the TEDIA Company Inc. (OH, USA). Other common chemicals and reagents were obtained rom the Shanghai Sangon Biotech Company (Shanghai, China). Milli-Q water was used in all experiments. The buffers used in this study are shown in Table 1.

\section{Aux/IAAs Clone and Recombinant Vector Construction}

Total RNA was extracted from 15-day-old Arabidopsis (Arabidopsis thaliana) by using Trizol reagent (Thermo Fisher Scientific Inc.) Reverse transcription was performed by using Script First-Strand cDNA Synthesis Super Mix Kit (TransGen Biotech, Beijing). Four pairs of primers were designed for recombinant vector construction based on the coding sequences of IAA1, IAA7 and IAA28 in Arabidopsis (Table 2). The amplification of full-length target genes was catalysed by high-fidelity DNA polymerase FastPFU (TransGen Biotech, Beijing) using cDNA as the template. The PCR products produced with the first three pairs of

Table 1 Buffers used in the study

\begin{tabular}{llc}
\hline Buffer & Components & pH value \\
\hline PBS & $140 \mathrm{mM} \mathrm{NaCl}, 2.7 \mathrm{mM} \mathrm{KCl}$, & 7.4 \\
& $10 \mathrm{mM} \mathrm{Na}_{2} \mathrm{HPO}_{4}$ and $1.8 \mathrm{mM} \mathrm{KH} \mathrm{PO}_{4}$ & \\
High pH buffer & $0.1 \mathrm{M} \mathrm{Tris-HCl}$ and $0.5 \mathrm{M} \mathrm{NaCl}$ & 8.5 \\
Low pH buffer & $0.1 \mathrm{M}$ sodium acetate, $0.5 \mathrm{M} \mathrm{NaCl}$ & 4.5 \\
Exchange buffer & $\begin{array}{l}50 \mathrm{mM} \text { Tris-HCl and } 10 \mathrm{mM} \\
\text { reduced glutathione }\end{array}$ & 8.5 \\
\hline
\end{tabular}


primers were inserted into the plasmid pGEX-KG after double digestion with SmaI/BamHI and EcoRI (Thermo Fisher Scientific Inc.). TAC promoter drove the expression of the target genes in E. coli. The PCR products were inserted into pIEx-3 by using IAA7-SF and IAA7-SR after their digestion by SalI and NotI (Thermo Fisher Scientific Inc.). IAA7 expression was driven by the IEI promoter with the $h r 5$ enhancer.

\section{Culture Conditions and Recombinant Protein Expression in E. Coli}

Expression vectors were transformed into three E. coli strains, including BL21, Tuner and Rosetta, by electroporation. Transformants were grown at $37{ }^{\circ} \mathrm{C}$ overnight in $\mathrm{LB}$ medium until the $\mathrm{OD}_{600}$ reached 0.6 . The cultures were diluted in fresh LB medium using a ratio of 1:50 and then grown in liquid culture at $37{ }^{\circ} \mathrm{C}$ on a shaker until the cells reached an $\mathrm{OD}_{600}$ of 0.6. Finally, the bacteria underwent another $5 \mathrm{~h}$ of shaking in culture to induce expression of the recombinant protein at $25{ }^{\circ} \mathrm{C}$ after adding IPTG to a concentration of $0.4 \mathrm{mM}$. The cells were collected by centrifugation and stored at $-80{ }^{\circ} \mathrm{C}$.

\section{Culture Conditions and Recombinant Protein Expression in $\mathbf{S 2}$}

Drosophila Schneider 2 (S2) derived from a primary culture of late stage Drosophila melanogaster embryos was purchased from Thermo Fisher Scientific Inc. (Catalogue no. R690-07). To increase the cell yield, the S2 cells were grown in Schneider's Drosophila medium (Catalogue no. 11720-034) at $28{ }^{\circ} \mathrm{C}$ without $\mathrm{CO}_{2}$ in suspension with spinners and shake flasks according to current protocols. Split cells at a 1:2 to 1:5 ratio were diluted into new culture every 3 to 4 days when the cells reached a density of 2 to $4 \times 10^{6}$ cells $/ \mathrm{mL}$. This procedure maintained the $\mathrm{S} 2$ cells. The insect expression vector was stably cotransfected into S2 with pCoHygro using the calcium phosphate transfection method, and the transfectants were selected by $300 \mu \mathrm{g} / \mathrm{mL}$ hygromycin-B. Cell death was verified by Trypan blue staining. A 19:1 (w/w) ratio of

Table 2 Primer lists in vector constructions

\begin{tabular}{|c|c|c|}
\hline $\begin{array}{l}\text { Primer } \\
\text { name }\end{array}$ & Sequences & $\begin{array}{l}\text { Enzyme } \\
\text { site }\end{array}$ \\
\hline IAA1F & 5'-cccgggAATGGAAGTCACCAATGGGC-3' & Smal \\
\hline IAA1R & 5'-gaattcTCATAAGGCAGTAGGAGCTTCG-3' & EcoRl \\
\hline IAA7-BF & 5'-ggatcCATGATCGGCCAACTTATGAACC-3' & BamHI \\
\hline IAA7-BR & 5'-gaattcTCAAGATCTGTTCTTGCAGTAC-3' & EcoRl \\
\hline IAA28F & 5'-ggatccATGGAAGAAGAAAAGAGATTGG-3' & BamHI \\
\hline IAA28R & 5'-gaattcCTATTCCTTGCCATGTTITCTAG-3' & EcoRl \\
\hline IAA7-SF & 5'-gtcgacATGATCGGCCAACTTATGAACCTC-3' & Sall \\
\hline IAA7-SR & 5'-gcggccgcTCAAGATCTGTTCTTGCAGTACTTC-3' & Notl \\
\hline
\end{tabular}

expression vector to selection vector was used in cotransfection. Target recombinant protein was secreted into the medium and then collected by centrifugation for further purification.

\section{Recombinant Protein Isolation and Purification}

Bacterial cells were collected and resuspended in PBS (pH 7.4). Cells were disrupted by ultrasonification. Supernatant was collected for target protein purification after centrifugation at 20,000 g for $10 \mathrm{~min}$ at $4{ }^{\circ} \mathrm{C}$. For insect expression, the medium was collected from $100-\mathrm{mL}$ cultures by centrifugation, since the recombinant protein was secreted into the medium. Supernatant was concentrated to less than $5 \mathrm{~mL}$ by centrifugation at $7500 \mathrm{~g}$ for 10 min in Amicon Ultra-4 Centrifugal Filter Devices with a $50 \mathrm{kDa}$ nominal molecular weight limit (NMWL) from Millipore at $4{ }^{\circ} \mathrm{C}$. Condensed culture medium was diluted 5 times in PBS to prepare it for protein purification. Protein solution was added to a column pre-filled with GST Sefirose $^{\mathrm{TM}}$ resin at a speed of $1 \mathrm{~mL} / \mathrm{min}$. The purified target proteins remained trapped in the column after it was washed with 5 resin volumes of PBS.

\section{IAA Quantification by LC-MS/MS}

Solution containing IAA was pumped into the column, and the IAA was trapped by the recombinant Aux/IAA proteins. Unbound molecules were washed off with 3 resin volumes of PBS. The outlet solution was collected after elution and freeze dried. The IAA sample was injected into LC-MS/MS (Shimadzu 8030 Plus) using a reverse $\mathrm{C} 18$ column to perform IAA determination after re-dissolving the compound in acetonitrile. Condition setting and programming for IAA detection were performed as described by Ma et al. [46].

\section{Results}

Expression of Aux/IAA Proteins in E. Coli

In this research, the recombinant vector was constructed based on the expression plasmid pGEX-KG with a GST tag (Fig. 1a) that enabled the convenient isolation and purification of recombinant proteins using GST resin. The expression level for a particular gene in prokaryotic expression is largely dependent on the strain. To ensure that an adequate level of recombinant protein was produced, the vector containing IAA1, IAA7 and IAA28 segments was transformed into three expression strains of $E$. coli, and the expression was compared in BL21, Tuner and Rosetta (Fig. 1b-d). IAA1, IAA7 and IAA28 were all highly expressed in different strains when they were induced by $0.4 \mathrm{mM}$ IPTG at $25{ }^{\circ} \mathrm{C}$. The results showed that the Rosetta strain was more effective at expressing IAA1 and IAA28, while Tuner expressed the highest levels of IAA7. The cells produced 0.019 (IAA1), 0.016 (IAA7) and 0.018 (IAA28) gram of recombinant 


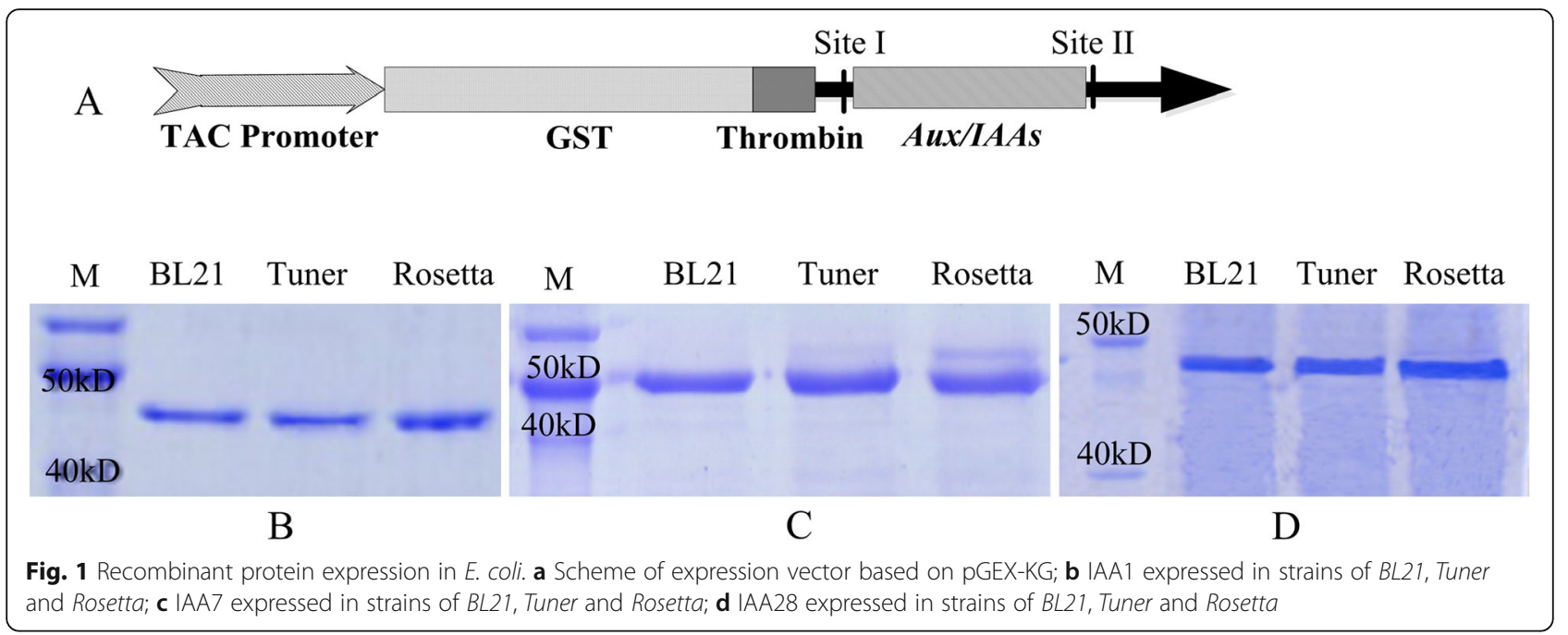

protein per gram of bacterial cells. Finally, we collected sufficient amounts of the recombinant proteins of IAA1, IAA7 and IAA28 from the cell supernatant using GST resin. No foreign bands existed after PAGE analysis (Fig. 1b-d), indicating that the highly purified protein can be used to analyse IAA binding.

\section{Expression of IAA7 Protein in S2 Cells}

In addition to prokaryotic cells, yeast, insect, mammalian and plant cells are frequently used to express recombinant proteins. Differences in protein processing, modification and folding after translation in different expression systems may influence the activity of the recombinant protein. To clarify whether the expression system affects the IAA binding characteristics of recombinant IAA7 protein, we optimized the culture conditions of S2 to be more suitable for the expression of IAA7 (Fig. 2b-j). The S2 cell line was derived from a primary culture of late-stage (20-24 h old) Drosophila melanogaster embryos. Many features of the S2 cell line suggest that it is derived from a macrophage-like lineage [47]. S2 cells can grow at room temperature without $\mathrm{CO}_{2}$ as a loose, semiadherent monolayer in tissue culture flasks and in suspension culture with spinners and shake flasks. In our experiment, the S2 cells grew better in spinners (Fig. 2b and c). Drosophila Schneider 2 cells can be transfected with the recombinant expression vector alone for transient expression studies or in combination with a selection vector to generate stable cell lines. We tested the expression of IAA7 protein by transient transfection before undertaking the selection of stable cell lines. The results proved the feasibility of IAA7 expression in S2. To create stable transfectants for longterm storage, increased expression and large-scale production of the desired protein, we employed the selection vector pCoHygro to perform co-transfection with pIEx-3-
IAA7 (Fig. 2a) and achieved stable cell lines by screening with hygromycin (Fig. 2d-i). For the final step, $5 \mathrm{mg}$ recombinant protein was purified from $100 \mathrm{~mL}$ culture medium through GST resin (Fig. 2j). The recombinant protein (approximately $64 \mathrm{kD}$ ) was a fusion protein including N-terminal GST-Tag, His-Tag and S-Tag and adiopkinetic hormone $(\mathrm{AKH})$ signal peptides that helped the recombinant protein to be secreted into the extracellular medium with high efficiency (Fig. 2a). In this study, we transfected secretion vector pIEx-3 into S2 and successfully screened a stable transfectant line. The results showed that pIEx-3 functioned effectively in Drosophila Schneider 2 cells, and the yield of the target recombinant protein was up to $5 \mathrm{mg}$ per $100 \mathrm{~mL}$ culture in the stable transfectant line.

\section{IAA Extraction and Purification}

In this study, we designed an IAA extraction and purification strategy based on the use of recombinant Aux/ IAA proteins. First, we pre-filled a cartridge with GST resin to selectively bind the GST-Aux/IAA fusion proteins. This gravity column permitted isolation and purification of the target recombinant protein from the cell supernatant, as GST resin showed ideal binding activity to bioactive GST fusion proteins. Next, the aqueous sample was passed through the gravity column, and the IAA was trapped by the Aux/IAA proteins. Purified IAA solution was collected and directly used to perform IAA determination by LC-MS/MS after elution. The gravity column filled with GST resin could be reused after regeneration.

To obtain the greatest amount of recovery, the ideal procedure would elute all of the trapped IAA from the gravity column with a limited volume of eluant as quickly as possible. To test the efficiency of the eluants, we selected five solutions as potential eluants, including ultra-pure water, $\mathrm{PBS}$, high $\mathrm{pH}$ buffer (HPB), low $\mathrm{pH}$ 

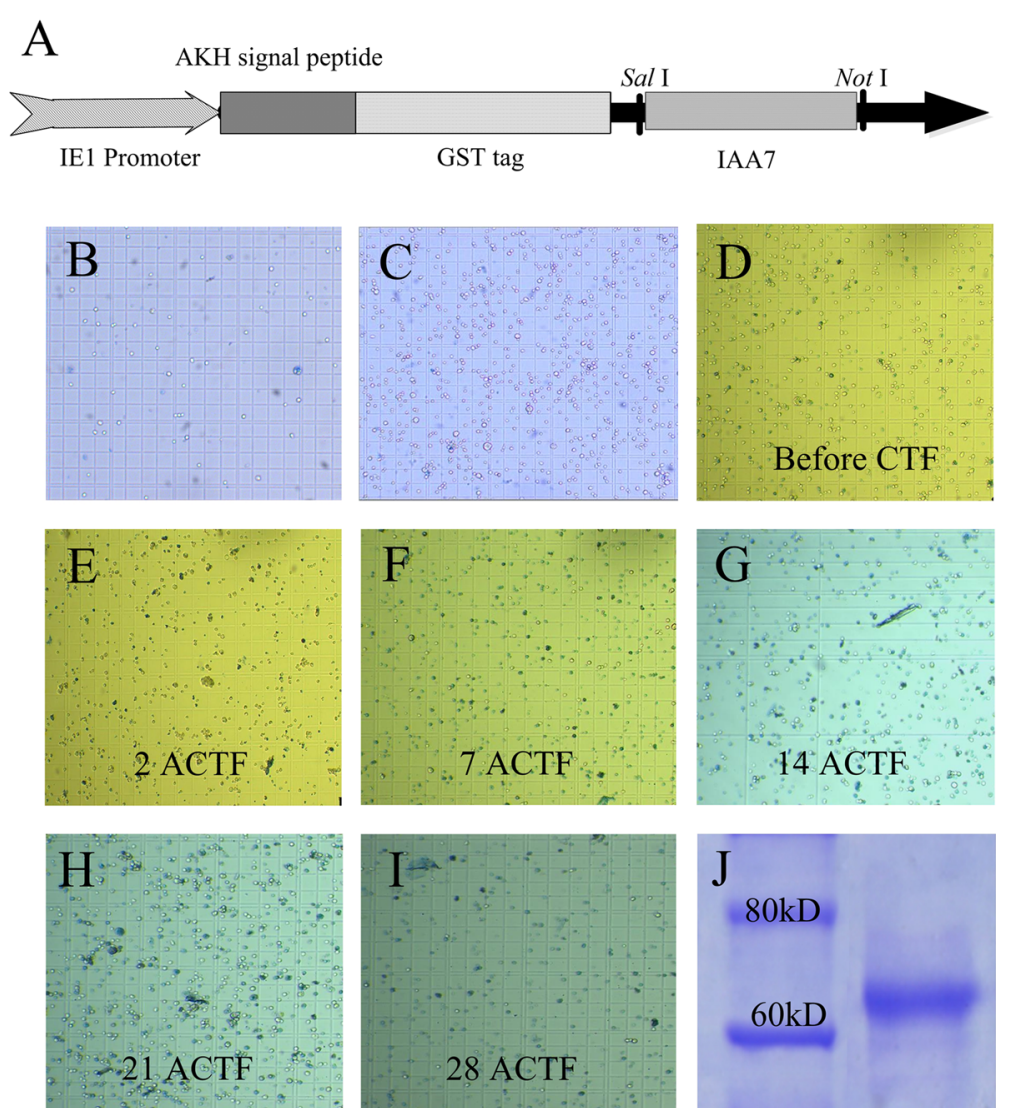

Fig. 2 Expression of recombinant IAA7 protein in S2. a Scheme of expression vector based on plEx-3; $\mathbf{b} 7 \mathrm{~d}$ adherent culture after passage; $\mathbf{c} 7 \mathrm{~d}$ suspension culture after passage; $\mathbf{d}$ S2 cells before co-transfection; e-i Stable cell line screening through homomycin after co-transfection (ACTF); j Gel analysis of target protein after purification from cell culture medium

buffer (LPB) and GST exchange buffer (reduced glutathione, RG). Eluants of water, and particularly PBS, stabilized the protein structure and maintained bioactivity. LC-MS/MS analysis indicated that little IAA remained in solution when water and PBS ( $\mathrm{pH}$ 7.4) were used to elute IAA from the extraction and purification column. In contrast, relatively higher alkaline or lower acid environments strongly affected the binding capability between GST and GST resin, or between Aux/IAA protein and IAA. The isoelectric points (pI) of IAA1, IAA7 and IAA28 are all greater than 7.0, thus indicating that their structures and bioactivities are more sensitive to alkaline conditions. Therefore, the results indicated that HPB ( $\mathrm{pH}$ 8.4) could more efficiently elute the IAA from the gravity column than LPB ( $\mathrm{pH} 4.5)$. GST exchange buffer containing $0.1 \mathrm{M}$ Tris- $\mathrm{HCl}$ and $10 \mathrm{mM}$ reduced glutathione was used to elute the expressed GST peptides from the GST resin (Fig. 3a). The eluting efficiency of the GST exchange buffer did not differ significantly from that of HPB (Fig. 3a). To simplify the process, the eluant was reduced to as small a volume as possible. In this research, IAA was almost entirely released from column after elution with 3 column volumes of HPB, LPB or RG
(Fig. 3b). In this method, the outlet solution contained only a few types of molecules and ions, including IAA, recombinant protein, Tris- $\mathrm{HCl}$ and $\mathrm{NaCl}$. Thus, the outlet solution is suitable for quantification by HPLCMS/MS. Therefore, this method greatly facilitates the sample preparation for tandem mass spec-based IAA quantification.

The different Aux/IAA proteins showed varying affinities towards auxin. To identify the Aux/IAA protein with the highest IAA binding capability, we added $1 \mathrm{ng}$ ${ }^{2} \mathrm{H}_{5}$-IAA dissolved in $5 \mathrm{~mL}$ PBS into the gravity column containing purified GST-IAA1, GST-IAA7 or GST-IAA28 protein derived from E. coli. The IAA was eluted by HPB after $10 \mathrm{~min}, 30 \mathrm{~min}$ and $60 \mathrm{~min}$ incubation at $4{ }^{\circ} \mathrm{C}$. The IAA in the outlet solution was measured using HPLCMS/MS (Fig. 4a). The standard curve (Fig. 4b) was used to calculate the contents of IAA after different types of outlet solutions were examined (Fig. 4c). The results indicated that the incubation time was critical for IAA to bind the Aux/IAA proteins (Fig. 4c). Their binding capabilities continually decreased as the incubation time was extended. In addition, IAA7 showed higher affinity and binding efficiency for IAA when compared with IAA1 and 


\begin{tabular}{|c|c|c|c|}
\hline Eluents & pH value & Efficiency & $\begin{array}{l}\text { IAA sample } \\
\text { preparation }\end{array}$ \\
\hline Water & 7.0 & - & - \\
\hline PBS & 7.4 & - & - \\
\hline HPB & 8.5 & +++ & easy \\
\hline LPB & 4.5 & + & easy \\
\hline RG & 8.5 & +++ & easy \\
\hline
\end{tabular}

\begin{tabular}{|c|c|c|c|c|c|c|}
\hline \multirow{2}{*}{ Eluents } & \multicolumn{6}{|c|}{ Volume of eluent versus volume of column } \\
\cline { 2 - 8 } & $\mathbf{0 . 5}$ Time & $\mathbf{1}$ Time & $\mathbf{2}$ Time & $\mathbf{3}$ Time & 4 Time & $\mathbf{5}$ Time \\
\hline Water & - & - & - & - & - & - \\
\hline PBS & - & - & - & - & - & - \\
\hline HPB & + & ++ & +++ & ++++ & ++++ & ++++ \\
\hline LPB & + & + & ++ & ++ & +++ & ++++ \\
\hline RG & + & ++ & +++ & ++++ & ++++ & ++++ \\
\hline
\end{tabular}

Fig. 3 Eluting IAA through various eluents. a Efficiency of eluting IAA from column and difficulty level of IAA sample preparation before quantification after elution; $\mathbf{b}$ Efficiency of eluting IAA through various volumes of eluents
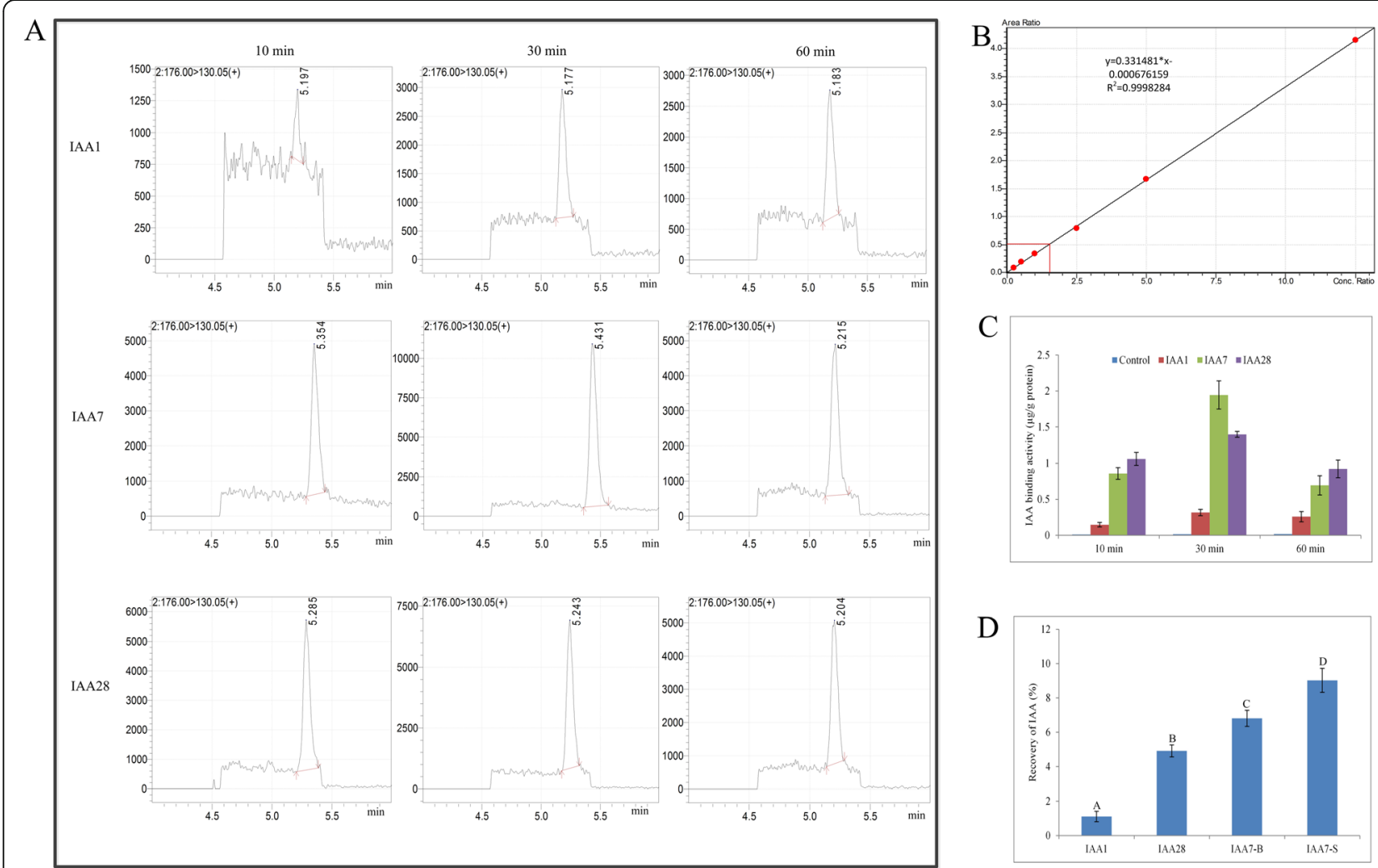

$\mathrm{C}$

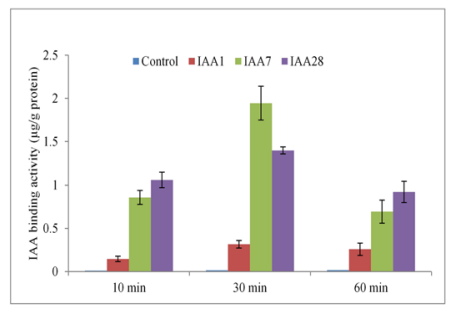

$\mathrm{D}$

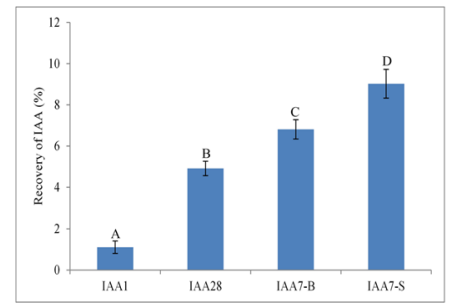

Fig. 4 IAA extraction and purification through Aux/IAA proteins. a IAA determination through LC-MS/MS; b Standard curve of IAA; c IAA binding activities of Aux/IAA protein after $10 \mathrm{~min}, 30 \mathrm{~min}$ and $60 \mathrm{~min}$ incubation in column, and control was the column containing GST resin but no recombinant proteins; $\mathbf{d}$ IAA recoveries when using IAA1 (expressed in Rosetta), IAA7 (expressed in Tuner and S2 cells) and IAA28 (expressed in Rosetta) as the sorbents 
IAA28. We also compared the difference in binding capability between IAA7 expressed in bacterial cells (IAA7-B) and that expressed in S2 (IAA7-S) (Fig. 4d). The result showed that the bioactivity of IAA7-S was significantly higher than that of IAA7-B (Fig. 4d). This result indicated that the binding capability of the recombinant protein towards IAA was higher when expressed in eukaryotes.

\section{Discussion}

\section{S2-plEx-3 System is Favourable for Expression of Higher Bioactivity Aux/IAA Proteins}

The recombinant proteins derived from various expression systems displayed differing amounts of bioactivity, and the pIEx-3 was suitable for expressing highly bioactive proteins. The pIEx-3 vector was previously designed for the cloning and expression of proteins in transiently transfected Spodoptera-derived insect cells. Transcription is driven by the AcNPV-derived $h r 5$ enhancer and the immediate early promoter $I E 1$. pIEx-3 contains the coding sequence for the signal peptide of adipokinetic hormone $(\mathrm{AKH})$ to allow the secretion of the expressed protein $[48,49]$. pIEx-3 also contains the $\mathrm{N}$-terminal GST-Tag, His-Tag, and S-Tag, as well as a C-terminal HSV-Tag coding sequence for protein detection and purification (Fig. 2a). More than 40 proteins, including cytoplasmic protein kinases or regions of a receptor with kinase activity, kinase interacting proteins, phospolipases, nuclear transport proteins, phosphatases, and heat shock proteins have been expressed using this InsectDirect System, and the yields were as high as $8 \mathrm{mg}$ from $100 \mathrm{~mL}$ culture in Sf9 and Sf21 cells [50]. In this study, the recombinant proteins displayed higher bioactivity, although lower recombinant protein yield was obtained in the stable transfectant S2 line (Fig. 4d). One potential reason for this could be that the posttranslation, modification and folding in insects may differ from these processes in native plants.

\section{The Aux/IAA Proteins Exhibited Different IAA Affinity}

Aux/IAA proteins belong to a large protein family. In Arabidopsis, 29 Aux/IAA proteins have been identified, and the existence of multiple Aux/IAA-ARF combinations may mediate specific responses [51-54]. The type of Aux/IAA protein may be the key factor in the auxin trapping that occurs in plant cells, since different Aux/ IAA-TIR1 co-receptors varied greatly in their affinity for auxin [45]. In this study, the higher IAA affinity observed with the IAA7 protein confirms this hypothesis.

\section{Affinity IAA Extraction and Purification Methods Showed Great Potential}

The ideal extraction and purification method for a particular phytohormone must be simple, rapid and specific to reduce its degradation and improve its recovery, as plant scientists require accurate quantification in trace plant tissues. It is critical to keep developing new extraction and purification methods for these compounds to satisfy the need for greater precision that accompanies the highly active field of investigation into the mechanisms of phytohormone actions. Currently, organic extraction and solid-phase extraction are the most frequently used methods in phytohormone extraction and purification prior to their analysis by LC-MS/MS. MIPs and some developed operational strategies, such as two-dimensional HPLC, online 2D HPLC and high-performance thin-layer chromatography (HPTLC), have been employed to purify phytohormones $[23,55,56]$.

To further simplify the extraction procedure and improve specificity for a particular phytohormone, the use of bioactive protein/complex applications shows great potential in phytohormone sample preparation. For example, some functional proteins in the signalling pathway of auxin, such as auxin binding proteins, receptors and co-receptors, are able to accurately recognize and bind auxin molecules even below the fM level in plant cells. Thus, Aux/IAA proteins display a high level of affinity and selectivity towards IAA. In this study, Aux/IAA proteins were used as a selective sorbent, and an affinity IAA extraction and purification method was developed. This method eliminated the problem of interference by structurally similar compounds and simplified the procedure of IAA extraction and purification from plant crude extracts. Our application of Aux/IAA protein validated the use of phytohormonal signalling proteins in phytohormone extraction and purification.

IAA can be extracted and purified from crude sample extracts using our method, but unfortunately not all of the protein was recovered. One reason for this could be the limited bioactivity and stability of the Aux/IAA proteins. To achieve highly stable Aux/IAA proteins, other expression systems should be tested to produce recombinant proteins; other tags should be used to solidify target proteins, and other buffers should be tested for the isolation and purification of proteins and the trapping of IAA. To achieve higher binding capabilities, the IAA co-receptor, which complexes with both the TIR1 and Aux/IAA protein, should be further tested as a sorbent since this co-receptor displayed a much greater affinity for auxin [45].

\section{Conclusion}

We expressed Aux/IAA proteins in E. coli and S2 cells and developed a method for IAA extraction and purification based on the recombinant proteins of Aux/IAAs. This method can be used for reference in bioactivity study and detection practice for other bioactive molecules. 


\section{Additional file}

Additional file 1: Aux/IAAs cloning and sequencing. (PDF $500 \mathrm{~kb}$ )

\section{Abbreviations}

ARF: Auxin Response Factor; Aux/IAA: AUXIN RESISTANT/INDOLE-3-ACETIC ACID INDUCIBLE; CAN: Acetonitrile; DLLME: Dispersive Liquid-Liquid Microextraction; DLSPE: Double Layered SPE; HF-LLLME: Hollow Fiber-Based Liquid-Liquid-Liquid Microextraction; HPB: High pH Buffer; IAA: Indole-3Acetic Acid; IAC: Immunoaffinity Chromatography; IAG: Immunoaffinity Gel; LLE: Liquid-Liquid Extraction; LLME: Liquid-Liquid Microextraction; LPB: Low pH Buffer; MIPs: Molecularly Imprinted Polymers; PMME: Polymer Monolith Microextraction; RG: Reduced Glutathione; SPE: Solid-Phase Extraction; SPME: Solid-Phase Microextraction; TIR1/AFB: TRANSPORT INHIBITOR RESPONSE1/AUXIN SIGNALING F-BOX

\section{Acknowledgments}

This research was carried out with funding from National Natural Science Foundation of China and Scientific Research Fund of Hunan Provincial Education Department. We also wish to thank Professor Keqin Peng and Professor Ruozhong Wang for their valuable advises.

\section{Funding}

This work was financially supported by National Natural Science Foundation of China (Grant No. 91317312, 31570372 and 9141730003) and Scientific Research Fund of Hunan Provincial Education Department (Grant No. 13 K065 and $15 \mathrm{~K} 061$ ).

\section{Availability of Data and Materials}

DNA sequences used in this study and cloning data were available as Additional file. Additional file 1: S1 Aux/IAA sequences cloned in this paper. Additional file 1: S2 IAA1 cloning and sequencing. Additional file 1: S3 IAA7 cloning and sequencing. Additional file 1: S4 IAA28 cloning and sequencing.

\section{Authors' Contributions}

$L X$ and $Y S$ designed the project, analyzed data and wrote the manuscript YS, WL, XC, HL and YH conducted the experiments. WL analyzed the data and revised the manuscript. All authors review and approved the final manuscript.

\section{Competing Interests}

The authors declare that they have no competing interests.

\section{Consent for Publication}

Not applicable.

\section{Ethics Approval and Consent to Participate} Not applicable.

Received: 24 June 2016 Accepted: 12 December 2016

Published online: 13 January 2017

\section{References}

1. Rademacher EH, Lokerse AS, Schlereth A, Llavata-Peris Cl, Bayer M, Kientz M, et al. Different Auxin Response Machineries Control Distinct Cell Fates in the Early Plant Embryo. Dev Cell. 2012;22:211-22.

2. Saini S, Sharma I, Kaur N, Pati PK. Auxin: A Master Regulator in Plant Root Development. Plant Cell Rep. 2013:32:741-57.

3. Kojima M, Kamada-Nobusada T, Komatsu H, Takei K, Kuroha T, Mizutani M, et al. Highly Sensitive and High-Throughput Analysis of Plant Hormones Using MS-Probe Modification and Liquid Chromatography-Tandem Mass Spectrometry: An Application for Hormone Profiling in Oryza Sativa. Plant Cell Physiol. 2009;50:1201-14.

4. Trapp MA, De Souza GD, Rodrigues-Filho E, Boland W, Mithöfer A. Validated Method for Phytohormone Quantification in Plants. Front Plant Sci. 2014;5:417.

5. Ljung K, Sandberg G, Moritz T. Hormone analysis. In: Davies PJ, editor. Plant hormones: biosynthesis, signal transduction, action. 3rd ed. Dordrecht/ Boston/London: Kluwer Academic Publishers; 2004. p. 1-15.

6. Fu J, Sun X, Wang J, Chu J, Yan C. Progress in Quantitative Analysis of Plant Hormones. Chin Sci Bull. 2011;56:355-66.
7. Vine $\mathrm{JH}$, Noiton D, Plummer JA, Baleriola-Lucas C, Mullins MG. Simultaneous Quantitation of Indole 3-Acetic Acid and Abscisic Acid in Small Samples of Plant Tissue by gas Chromatography/Mass Spectrometry/Selected ion Monitoring. Plant Physiol. 1987:85:419-22.

8. Engelberth J, Schmelz EA, Alborn HT, Cardoza YJ, Huang J, Tumlinson JH. Simultaneous Quantification of Jasmonic Acid and Salicylic Acid in Plants by Vapor-Phase Extraction and gas Chromatography-Chemical Ionization-Mass Spectrometry. Anal Biochem. 2003;312:242-50.

9. Ross AR, Ambrose SJ, Cutler AJ, Feurtado JA, Kermode AR, Nelson K, et al. Determination of Endogenous and Supplied Deuterated Abscisic Acid in Plant Tissues by High-Performance Liquid Chromatography-Electrospray Ionization Tandem Mass Spectrometry With Multiple Reaction Monitoring. Anal Biochem. 2004;329:324-33.

10. Vilaró F, Canela-Xandri A, Canela R. Quantification of Abscisic Acid in Grapevine Leaf (Vitis Vinifera) by Isotope-Dilution Liquid ChromatographyMass Spectrometry. Anal Bioanal Chem. 2006;386:306-12.

11. Novák O, Hényková E, Sairanen I, Kowalczyk M, Pospíšil T, Ljung K. TissueSpecific Profiling of the Arabidopsis Thaliana Auxin Metabolome. Plant J. 2012:72:523-36.

12. Barkawi LS, Tam YY, Tillman JA, Normanly J, Cohen JD. A High-Throughput Method for the Quantitative Analysis of Auxins. Nat Protoc. 2010:5:1609-18.

13. Li G, Lu S, Wu H, Chen G, Liu S, Kong X, et al. Determination of Multiple Phytohormones in Fruits by High-Performance Liquid Chromatography With Fluorescence Detection Using Dispersive Liquid-Liquid Microextraction Followed by Precolumn Fluorescent Labeling. J Sep Sci. 2015:38:187-96.

14. HEMBERG T, WESTLIN PE. The Quantitative Yield in Purification of Cytokinins. Model-Experiments With Kinetin, 6-Furfuryl-Amino-Purine. Physiol Plant. 1973;28:228-31.

15. Schmelz EA, Engelberth J, Alborn HT, O'Donnell P, Sammons M, Toshima H, et al. Simultaneous Analysis of Phytohormones, Phytotoxins, and Volatile Organic Compounds in Plants. Proc Natl Acad Sci U S A. 2003;100:10552-7.

16. Durgbanshi A, Arbona V, Pozo O, Miersch O, Sancho JV, Gómez-Cadenas A. Simultaneous Determination of Multiple Phytohormones in Plant Extracts by Liquid Chromatography-Electrospray Tandem Mass Spectrometry. J Agric Food Chem. 2005;53:8437-42.

17. Xie W, Han C, Zheng Z, Chen X, Qian Y, Ding H, et al. Determination of Gibberellin A3 Residue in Fruit Samples by Liquid Chromatography-Tandem Mass Spectrometry. Food Chem. 2011;127:890-2.

18. Wu Y, Hu B. Simultaneous Determination of Several Phytohormones in Natural Coconut Juice by Hollow Fiber-Based Liquid-Liquid-Liquid Microextraction-High Performance Liquid Chromatography. J Chromatogr. 2009:1216:7657-63.

19. Lu Q, Zhang W, Gao J, Lu M, Zhang L, Li J. Simultaneous Determination of Plant Hormones in Peach Based on Dispersive Liquid-Liquid Microextraction Coupled With Liquid Chromatography-ion Trap Mass Spectrometry. J Chromatogr B. 2015;992:8-13.

20. Gupta V, Kumar M, Brahmbhatt H, Reddy C, Seth A, Jha B. Simultaneous Determination of Different Endogenetic Plant Growth Regulators in Common Green Seaweeds Using Dispersive Liquid-Liquid Microextraction Method. Plant Physiol Biochem. 2011;49:1259-63.

21. Poole CF. New Trends in Solid-Phase Extraction. TrAC Trends Anal Chem. 2003;22:362-73.

22. Podlesakova K, Tarkowska D, Pencik A, Oklestkova J, Tureckova V, Flokova K, et al. New Trends in Phytohormone Analysis. Chem Listy. 2012;106:373-9.

23. Dobrev $P$, Havlíček L, Vágner M, Malbeck J, Kamínek M. Purification and Determination of Plant Hormones Auxin and Abscisic Acid Using Solid Phase Extraction and two-Dimensional High Performance Liquid Chromatography. J Chromatogr. 2005;1075:159-66.

24. Liu JF, Ding J, Yuan BF, Feng YQ. Magnetic Solid Phase Extraction Coupled With in Situ Derivatization for the Highly Sensitive Determination of Acidic Phytohormones in Rice Leaves by UPLC-MS/MS. Analyst. 2014;139:5605-13.

25. Floková K, Tarkowská D, Miersch O, Strnad M, Wasternack C, Novák O. UHPLC-MS/MS Based Target Profiling of Stress-Induced Phytohormones. Phytochemistry. 2014;105:147-57.

26. Cui K, Lin Y, Zhou X, Li S, Liu H, Zeng F, et al. Comparison of Sample Pretreatment Methods for the Determination of Multiple Phytohormones in Plant Samples by Liquid Chromatography-Electrospray Ionization-Tandem Mass Spectrometry. Microchem J. 2015;121:25-31.

27. Liu HT, Li Y-F, Luan TG, Lan CY, Shu WS. Simultaneous Determination of Phytohormones in Plant Extracts Using SPME and HPLC. Chromatographia. 2007:66:515-20 
28. Ding J, Mao LJ, Wang ST, Yuan BF, Feng YQ. Determination of Endogenous Brassinosteroids in Plant Tissues Using Solid-Phase Extraction With Double Layered Cartridge Followed by High-Performance Liquid ChromatographyTandem Mass Spectrometry. Phytochem Anal. 2013;24:386-94.

29. Hennion MC. Solid-Phase Extraction: Method Development, Sorbents, and Coupling With Liquid Chromatography. J Chromatogr. 1999;856:3-54.

30. Khan A, Khan Ml, labal Z, Ahmad L, Shah Y, Watson DG. Determination of Lipoic Acid in Human Plasma by HPLC-ECD Using Liquid-Liquid and SolidPhase Extraction: Method Development, Validation and Optimization of Experimental Parameters. J Chromatogr B. 2010;878:2782-8.

31. Risticevic S, Lord H, Górecki T, Arthur CL, Pawliszyn J. Protocol for SolidPhase Microextraction Method Development. Nat Protoc. 2010;5:122-39.

32. Chen C, Chen Y, Zhou J, Wu C. A 9-Vinyladenine-Based Molecularly Imprinted Polymeric Membrane for the Efficient Recognition of Plant Hormone 1 H-Indole-3-Acetic Acid. Anal Chim Acta. 2006;569:58-65.

33. Ding J, Mao $L$ J, Yuan BF, Feng YQ. A Selective Pretreatment Method for Determination of Endogenous Active Brassinosteroids in Plant Tissues: Double Layered Solid Phase Extraction Combined With Boronate Affinity Polymer Monolith Microextraction. Plant Methods. 2013;9:1.

34. Porobić I, Kontrec D, Šoškić M. Molecular Recognition of Indole Derivatives by Polymers Imprinted With Indole-3-Acetic Acid: A QSPR Study. Biorg Med Chem. 2013;21:653-9.

35. Tamayo F, Turiel E, Martín-Esteban A. Molecularly Imprinted Polymers for Solid-Phase Extraction and Solid-Phase Microextraction: Recent Developments and Future Trends. J Chromatogr. 2007;1152:32-40.

36. Turiel E, Martín-Esteban A. Molecularly Imprinted Polymers for Solid-Phase Microextraction. J Sep Sci. 2009;32:3278-84.

37. Polyanina D, Beklemishev M. Molecularly Imprinted Inorganic Supports in High-Performance Liquid Chromatography and Solid-Phase Extraction. J Anal Chem. 2015;70:277-86.

38. Hauserová E, Swaczynová J, Doležal K, Lenobel R, Popa I, Hajdúch M, et al. Batch Immunoextraction Method for Efficient Purification of Aromatic Cytokinins. J Chromatogr. 2005;1100:116-25.

39. Hradecká V, Novák O, Havlíček L, Strnad M. Immunoaffinity Chromatography of Abscisic Acid Combined With Electrospray Liquid Chromatography-Mass Spectrometry. J Chromatogr B. 2007;847:162-73.

40. López-Carbonell M, Gabasa M, Jáuregui O. Enhanced Determination of Abscisic Acid (ABA) and Abscisic Acid Glucose Ester (ABA-GE) in Cistus Albidus Plants by Liquid Chromatography-Mass Spectrometry in Tandem Mode. Plant Physiol Biochem. 2009;47:256-61.

41. Tarkowski P, Ge L, Yong JWH, Tan SN. Analytical Methods for Cytokinins. TrAC Trends Anal Chem. 2009;28:323-35.

42. Liang Y, Zhu X, Zhao M, Liu H. Sensitive Quantification of Isoprenoid Cytokinins in Plants by Selective Immunoaffinity Purification and High Performance Liquid Chromatography-Quadrupole-Time of Flight Mass Spectrometry. Methods. 2012;56:174-9.

43. Chapman EJ, Estelle M. Mechanism of Auxin-Regulated Gene Expression in Plants. Annu Rev Genet. 2009;43:265-85.

44. Enders TA, Strader LC. Auxin Activity: Past, Present, and Future. Am J Bot. 2015;102:180-96.

45. Villalobos LIAC, Lee $\mathrm{S}$, De Oliveira C, Ivetac A, Brandt W, Armitage L, et al. A Combinatorial TIR1/AFB-Aux/IAA co-Receptor System for Differential Sensing of Auxin. Nat Chem Biol. 2012;8:477-85.

46. Ma C, Meir S, Xiao L, Tong J, Liu Q, Reid M, Jiang C. A KNOTTED1-LIKE HOMEOBOX Protein Regulates Abscission in Tomato by Modulating the Auxin Pathway. Plant Physiol. 2015;167:844-53.

47. Schneider I. Cell Lines Derived from Late Embryonic Stages of Drosophila Melanogaster. Development. 1972;27:353-65.

48. Rodems SM, Friesen PD. The hr5 Transcriptional Enhancer Stimulates Early Expression from the Autographa Californica Nuclear Polyhedrosis Virus Genome but is not Required for Virus Replication. J Virol. 1993; 67:5776-85

49. Guarino LA, Dong W. Expression of an Enhancer-Binding Protein in Insect Cells Transfected With the Autographa Californica Nuclear Polyhedrosis Virus IE1 Gene. J Virol. 1991;65:3676-80.

50. Jarvis DL, Weinkauf C, Guarino LA. Immediate-Early Baculovirus Vectors for Foreign Gene Expression in Transformed or Infected Insect Cells. Protein Expression Purif. 1996:8:191-203.

51. Remington DL, Vision TJ, Guilfoyle TJ, Reed JW. Contrasting Modes of Diversification in the Aux/IAA and ARF Gene Families. Plant Physiol. 2004;135:1738-52.
52. Overvoorde PJ, Okushima Y, Alonso JM, Chan A, Chang C, Ecker JR, et al. Functional Genomic Analysis of the AUXIN/INDOLE-3-ACETIC ACID Gene Family Members in Arabidopsis Thaliana. Plant Cell. 2005;17:3282-300.

53. Krogan NT, Ckurshumova W, Marcos D, Caragea AE, Berleth T. Deletion of MP/ARF5 Domains III and IV Reveals a Requirement for Aux/IAA Regulation in Arabidopsis Leaf Vascular Patterning. New Phytol. 2012;194:391-401.

54. Krogan NT, Yin X, Ckurshumova W, Berleth T. Distinct Subclades of AuX/IAA Genes are Direct Targets of ARF5/MP Transcriptional Regulation. New Phytol. 2014;204:474-83.

55. Zhong Q, Qiu X, Lin C, Shen L, Huo Y, Zhan S, et al. An Automatic Versatile System Integrating Solid-Phase Extraction With Ultra-High Performance Liquid Chromatography-Tandem Mass Spectrometry Using a Dual-Dilution Strategy for Direct Analysis of Auxins in Plant Extracts. J Chromatogr. 2014;1359:131-9.

56. Goswami D, Thakker JN, Dhandhukia PC. Simultaneous Detection and Quantification of Indole-3-Acetic Acid (IAA) and Indole-3-Butyric Acid (IBA) Produced by Rhizobacteria from l-Tryptophan (Trp) Using HPTLC. J Microbiol Methods. 2015;110:7-14.

\section{Submit your next manuscript to BioMed Central and we will help you at every step:}

- We accept pre-submission inquiries

- Our selector tool helps you to find the most relevant journal

- We provide round the clock customer support

- Convenient online submission

- Thorough peer review

- Inclusion in PubMed and all major indexing services

- Maximum visibility for your research

Submit your manuscript at www.biomedcentral.com/submit
C Biomed Central 